\title{
Effect of low dose ionizing radiation versus human wharton's jelly mesenchymal stem cells in the treatment of experimental rheumatoid arthritis
}

\author{
Doaa K. Sadek ${ }^{1}$, Monda M. M. Badawy ${ }^{2}$, Doaa M. Abd El-latif ${ }^{3}$ and Soheir S. Korraa ${ }^{2, *}$ \\ ${ }^{1}$ E xperimental and Advanced Pharmaceutical Research Unit, Faculty of Pharmacy, Ain Shams University, Cairo, Egypt. \\ ${ }^{2}$ Department of Health Radiation Research, National Center for Radiation Research and Technology, Egyptian Atomic \\ Energy Authority, Cairo, Egypt. \\ ${ }^{3}$ Department of Biochemistry, Faculty of Pharmacy (Girls), Al-Azhar University, Cairo, Egypt. \\ *Correspondence: doaakaram@pharma.asu.edu.eg
}

Article history: Received 2020-12-16 Revised 2021-01-07 Accepted 2021-04-04

\begin{abstract}
Rheumatoid arthritis (RA) is one of the major causes of disability that aggrieve a financial burden for the healthcare system as well as for patients. Both stem cell therapy and low-dose radiotherapy (LD-RT) have been recognized as alternative approaches to alleviate pain, inflammation, and bone erosion in the inflamed joints. Accordingly, the present study was designed to compare the efficacy of both modalities in the treatment of RA in experimental rats. Rheumatoid arthritis was induced in forty female rats using Complete Freund's adjuvant (CFA), divided into four groups (arthritic induction group and three treatment groups) compared to controls: one of the treatment groups received Human's Wharton Jelly Mesenchymal Stem Cells (WJ-MSC), and the other two groups received individual doses of 0.5 and 1 Gy of Gamma irradiation once a week for six weeks. After six weeks animals were sacrificed and examined for joint histology, paw swelling, mRNA gene expression for TNF- $\alpha$, IL-1 $\beta$ and IL- 6 together with plasma anticyclic citrullinated peptide (anti$\mathrm{CCP}$ ) and nitric oxide (NO). Results showed that both doses of (0.5 Gy and $1 \mathrm{~Gy})$ and WJ-MSC individually modulated RA effects by significantly decreasing the measured biochemical and molecular parameters. However, at the histology level it was evident that irradiation has a better pronounced effect compared to WJMSC, which showed aggravated effects on joint erosion. The present study reports that both LD-RT and WJMSC significantly decreased anti-CCP, which is a specific marker for RA, however, WJ-MSC might have deliberate effects on the synovitis and arthritis.
\end{abstract}

Keywords: Anticyclic-citrullinated; Protein (anti-CCP); Ionizing Radiation; Mesenchymal Stem Cells; Rheumatoid Arthritis.

\section{INTRODUCTION}

Rheumatoid arthritis (RA) is the most known inflammatory arthritis, with $1 \%$ prevalence in the general population worldwide and progressing with the increasing age of the population ${ }^{1}$. It is one of the major causes of disability that aggrieve a financial burden for the healthcare system as well as for patients and has a significant impact on occupational and daily activities, as well as mortality ${ }^{2}$. It is characterized by chronic inflammation of the joints associated with high influx of activated inflammatory immune cells, followed by a progressive cartilage and bone damage that end up with chronic synovitis, bone erosion, and eventual deformity ${ }^{3,4}$. It is documented that neutrophil infiltration secretes free radicals including nitric oxide (NO) and pro-inflammatory cytokines such as tumor necrosis factor- $\alpha$ (TNF- $\alpha)$, interleukin-1 $\beta$ (IL-1 $\beta)^{5,6}$ and interleukin-6 (IL-6), IL-
17 and the IL-12/23 family in rheumatoid arthritis despite their attractiveness as targets ${ }^{7}$. Lately, Anticyclic citrullinated peptide (anti-CCP) has been evaluated in a cohort of patients with early synovitis and was found to be of specificity and sensitivity for $\mathrm{RA}$ diagnosis. The sensitivity of anti-CCP is 48$80 \%$, and specificity is $96-98 \%{ }^{8}$. The anti-CCP ELISA was shown to be highly specific for RA and can be detected in up to $35 \%$ of RF-negative sera ${ }^{9}$.

Efficient anti-rheumatic treatment regimens are available, however novel and/or alternative treatments would, therefore, be of value and more additional therapeutic options are needed for patients with initial high disease activity or early joint damage that do not respond to conventional treatment ${ }^{10,11}$. Accordingly, additional therapeutic options including stem cell therapy or low-dose radiotherapy (LD-RT) has been applied to attenuate inflammation and bone destruction.

Cite this article: Sadek, D., Badawy, M., Abd El-latif, D., Korraa, S. Effect of low dose ionizing radiation versus human wharton's jelly mesenchymal stem cells in the treatment of experimental rheumatoid arthritis. Azhar International Journal of Pharmaceutical and Medical Sciences, 2021; 1(2):30-39. doi: 10.21608/aijpms.2021.53417.1020

DOI: 10.21608 /aijpms.2021.53417.1020 
Recently, researchers are directed to umbilical cord derived wharton's Jelly cells as an emerging source of mesenchymal stromal cells (MSCs) for future cell-based therapy ${ }^{12}$. Umbilical cord mesenchymal stem cells (UC-MSC) exhibit special characteristics over other sources of MSC due to noninvasive isolation, ease of access with no ethical concern, and devoid of pro-aging factors for its neonatal origin ${ }^{13}$. In addition to these advantages, UC-MSCs retain immunoregulatory properties and hypo-immunogenicity of $\mathrm{MSCs}^{14}$. Other studies have reported the successful therapeutic potential of human UC-MSC in arthritic experimental animals ${ }^{15,16}$.

The efficiency of LD-RT has been reported in the treatment of degenerative musculoskeletal diseases and inflammatory benign disorders ${ }^{17}$. Administrating radiation therapy at low doses has been recognized as one of the approaches for the treatment of $\mathrm{RA}^{18}$. Several studies reported the antiinflammatory properties of LD-RT; however, underlying mechanisms are not well understood ${ }^{19,20}$.

Accordingly, the present study was designed to compare the efficacy of WJ-MSC and Low-dose gamma radiation modalities in the treatment of RA in experimental rats.

\section{METHODS}

\subsection{Animal model and induction of arthritis}

Fifty female albino rats (Rattus norvegicus) (6-8 weeks) weighing 140-160g were housed at the animal house-Faculty of Pharmacy-Ain Shams University. All experimental treatments were approved by the Research Ethics Committee at the Faculty of Pharmacy-Azhar University (approval no. 86/2016). Experimental animals were housed under controlled conditions for 5 days before any intervention. They were divided into 5 experimental groups:

Group I is the negative control,

Group II is the arthritic group with no treatment provided,

Group III is the Wharton's Jelly MSC treated group after arthritic induction,

Group IV is 0.5 Gy ionizing irradiation treated group after arthritic induction and

Group $\mathrm{V}$ is 1 Gy ionizing irradiation treated group after arthritic induction.

Rheumatoid arthritis will be induced in all groups except the negative control group. Animals were induced for rheumatoid arthritis by an intradermal injection of $0.5 \mathrm{ml}^{21}$ of Complete Freund's adjuvant (CFA) containing $1.0 \mathrm{mg}$ of dry, heat-killed Mycobacterium tuberculosis suspended in $0.85 \mathrm{ml}$ paraffin oil and $0.15 \mathrm{ml}$ of mannide monooleate, (Sigma-Aldrich) into the left hind footpad as described by Fletcher et al., $1998^{22}$. Negative control rats were injected with $0.5 \mathrm{ml}$ sterilized saline solution. Swelling and oedema were monitored, and the mediolateral joint diameter was measured using a vernier calliper to confirm success of disease induction. Full progression of the disease was reached after 10-14 days of CFA injection.

\subsection{Isolation and Culture of Human WJ-MSCs}

Human umbilical cords were obtained immediately after full-term birth. The umbilical cords were collected in cold phosphate buffered saline containing $100 \mathrm{U} / \mathrm{mL}$ penicillin, $100 \mu \mathrm{g} / \mathrm{mL}$ streptomycin and processed within 12 hours for isolation of WJ-MSC by using the described method by La Rocca et al., $2009^{23}$. Cords were cut into small pieces, arteries and veins were discarded, and amniotic epithelium was scraped. Then, umbilical cord matrix was cut into small explant pieces (about $4 \times 5 \mathrm{~mm}$ ). Explants were cultured in RPMI with stable glutamine (Biowest, USA) containing 10\% fetal bovine serum (Gibco, USA), and $100 \mathrm{U} / \mathrm{mL}$ penicillin, $100 \mu \mathrm{g} / \mathrm{mL}$ streptomycin were added after. The MSCs were passaged after reaching 70-80\% confluence. At passage 3 the cells were harvested and analyzed by flowcytometry for cell surface markers CD45, CD34, CD73, CD90, CD271.

\subsection{Treatment regimen}

Treatment began at day 14 of CFA injection, when arthritis had become well established. Group III was injected intravenously with Wharton's Jelly MSC at a dose of $2 \times 10^{6}$ cells while groups IV and V were exposed to whole body gamma radiation at doses of 0.5 or 1.0 Gy once every week for six weeks. The application of irradiation was performed at Egyptian Atomic Energy Authority. The dose rate of the caesium-137 was $0.685 \mathrm{rad} / \mathrm{sec}$. at the time of experimentation. Phosphate buffer saline was injected in the negative control and arthritic groups. Every 2 days, the paw swelling of the rats was examined. All rats were sacrificed 8 weeks after the CFA injection. Blood was then collected and placed in heparinized tubes. Plasma was prepared by centrifugation at 3000 rpm for $15 \mathrm{~min}$ and stored at $-70^{\circ} \mathrm{C}$. The spleens were removed, and stored at $-70^{\circ} \mathrm{C}$.

\subsection{Histopathological examination}

After scarification, the bone was removed and fixed in $10 \%$ neutral buffered formalin for $48 \mathrm{~h}$, and then decalcified in $10 \%$ ethylene diamine tetra-acetic acid 30 days. Tissue processing was performed by dehydration, clearing, impregnation, and embedding using graded ethanol, xylol, and paraffin. Histological sections were prepared with a thickness of $6 \mu \mathrm{m}$ taken from each defect containing an intact border of the bone, and then the samples were routinely stained with hematoxylin and eosin ${ }^{24}$. Inflammatory mononuclear cells infiltration, synovial hyperplasia, extension of pannus formation, synovial fibrosis, and cartilage erosion were examined in the joints of at 
least three animals in each group. Semi-quantitative scoring for the histological parameters was used according to Barsante et al., 2005 25 . Each of these parameters were given a score from zero to three ${ }^{26}$. Score zero represents the absence of the lesion, score 1 represents mild defect (1-10\%), score 2 represents moderate defect $(11-20 \%)$ and score 3 represents severe defect (51-100\%). For synovial hyperplasia, score zero represents the absence of hyperplasia, score 1 represents mild hyperplasia (5-10 layers), score 2 represents moderate hyperplasia (11-20 layers) and score 3 represents severe hyperplasia $((>20$ layers $)$.

\subsection{Measurement of Anticyclic-citrullinated protein in plasma (Anti-CCP)}

Quantitative determination of cyclic citrullinated protein autoantibody was performed by enzyme linked immunosorbent assay (ELISA) using MyBioSource ${ }^{\circledR}$ Rat Anti-Cyclic Cirtullinated Peptide Antibody (ACCPA) Elisa kit (Cat No.: MBS762429). The analysis was performed following the manufacturers procedure which is according to the principles illustrated by Lequin, $2005^{27}$.

\subsection{Determination of plasma nitric oxide (NO)}

Total nitrate $\backslash$ nitrite was measured in plasma as an index of nitric oxide (NO) production according to the method documented by Miranda et al., 200128.

\subsection{Real-time polymerase chain reaction (RT- PCR)}

To analyze gene expression of TNF $\alpha$, IL- $1 \beta$ and IL-6, total RNA was extracted from the collected homogenized spleens using RNeasy Mini Kit from QIAGEN Inc. (Cat No. 74104) according to the manufacturer's instructions. Then the sample was reverse transcribed for 30 minutes at $42^{\circ} \mathrm{C}$ using QuantiTect Reverse Transcription Kit from QIAGEN Inc. (Cat No./ ID: 205311) in the presence of oligo-dT primer. Quantitative real-time PCR was performed with Applied Biosystem StepOnePlus TM Real-Time PCR Systems using QuantiTect SYBER ${ }^{\circledR}$ Green PCR Kit (Cat No./ID: 204143). Specific primers for target genes were obtained from Invitrogen by life technologies ThermoFisher. Polymerase chain reaction was performed specific for each cDNA using specific primers from the published sequence shown in table 1. RT-PCR is performed according to principles described by Giulietti et al., $2001^{29}$.

\subsection{Statistical analysis}

All data is represented as mean values \pm SEM except for paw swelling dimensions represented as mean values $\pm \mathrm{SD}$. For statistical calculations we used one-way analysis of variance (ANOVA) followed by Tukey's multiple comparison test applied on GraphPad prism software version 5.01. Significant difference is considered at $\mathrm{P}$-value less than 0.05 .

\section{RESULTS}

\subsection{Isolation and characterization of Wharton's MSC}

WJ-MSCs grew in spindle shaped like cells and adhered to the walls. Flowcytometry analysis was used to detect surface marker of WJ-MSCs at passage 3. Results showed positive surface cell markers for CD73 and CD90 whereas negative surface cell markers for CD271, CD34, and CD45 as shown in Figure (1).

\subsection{Joint swelling measurement}

Hind paw swelling was assessed by measuring left joint circumference at the beginning of the study, after CFA arthritis induction and at the end of the study. Results show a highly significant increase in CFA induced arthritis paw joint swelling compared to controls $(\mathrm{p}<0.005)$. This swelling was decreased significantly in rat treated with $0.5 \mathrm{~Gy}, 1 \mathrm{~Gy}$ and WJMSC (Figures $2 \& 3$ ).

\subsection{Joint histology assessment}

Rat joints were assessed semi-quantitatively using five criteria which includes infiltrating mononuclear cells, synovial hyperplasia, extension of pannus formation, synovial fibrosis, and cartilage erosion. They were scored according to the previously mentioned scoring system. Results for semiquantitative scoring of the five experimental groups are shown in table 2. Normal structure in all histologic parameters was detected in the control group while in the CFA-arthritic group high scores were demonstrated for all the parameters. In the WJ-MSC treated group there was slight decrease in histological scores, but cartilage erosion was apparently decreased. The two irradiation groups demonstrated better histological scores compared to WJ-MSC. Photomicrographs for paw joint stained with hematoxylin and eosin (H\&EX200) are shown in Figure 4.

\subsection{Results of plasma Anti-CCP and NO in different groups}

Anti-CCP antibodies and NO were significantly increased in CFA-arthritic rats compared to controls, indicating that this study was successful in inducing RA in rats. However, treatment with WJ-MSC and both doses of ionizing radiation did not retrieve antiCCP antibodies to control level. (Figure 5). 
Sadek et al, Azhar Int J Pharm Med Sci 2021; Vol 1 (2):30-39

Table 1: Primer sequences for the genes amplified using quantitative R T-PCR.

\begin{tabular}{|c|c|c|c|c|c|}
\hline Gene & Strand & Sequence (5'-3') & $\begin{array}{l}\text { Gene Bank } \\
\text { accession \# }\end{array}$ & $\begin{array}{l}\text { Product } \\
\text { length } \\
\text { (bp) }\end{array}$ & $\begin{array}{c}\text { Annealing } \\
\text { temperature } \\
\left({ }^{\circ} \mathrm{C}\right)\end{array}$ \\
\hline \multirow[t]{2}{*}{ IL-6 } & $\mathrm{F}$ & CTCTCCGCAAGAGACTTCCAG & NM_012589.2 & 115 & 57 \\
\hline & $\mathrm{R}$ & TGTGGGTGGTATCCTCTGTGA & & & \\
\hline \multirow[t]{2}{*}{ IL-1 $\beta$} & $\mathrm{F}$ & GCAGGCTTCGAGATGAACAAC & NM_031512.2 & 142 & 55 \\
\hline & $\mathrm{R}$ & TTGTCGTTGCTTGTCTCTCCT & & & \\
\hline \multirow[t]{2}{*}{ TNF- $\alpha$} & $\mathrm{F}$ & GTGATCGGTCCCAACAAGGA & NM_012675.3 & 141 & 56 \\
\hline & $\mathrm{R}$ & CGCTTGGTGGTTTGCTACG & & & \\
\hline \multirow[t]{2}{*}{$\beta$-actin } & $\mathrm{F}$ & TTGTCCCTGTATGCCTCT & NM_031144.3 & 220 & 52 \\
\hline & $\mathrm{R}$ & TAATGTCACGCACGATTTCC & & & \\
\hline
\end{tabular}
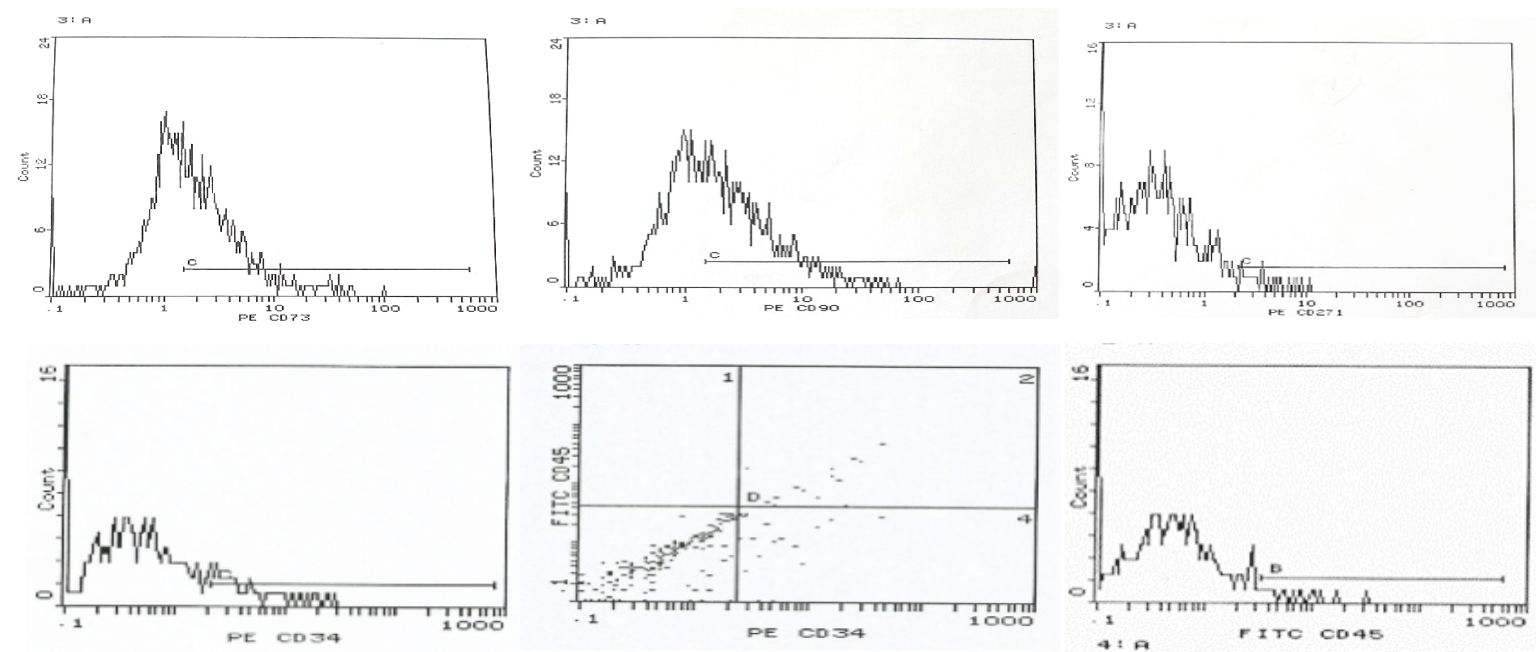

Figure 1. Characterization of Wharton's MSC. The flowcytometry analysis showing that WJ-MSCs were positive for CD73 and CD90 whereas negative for CD271, CD34 and CD45.
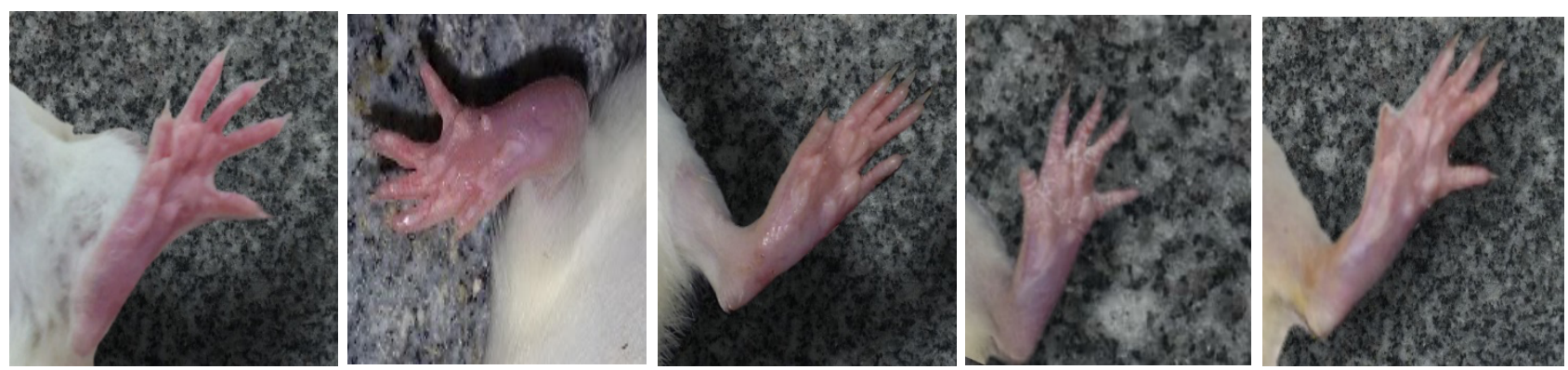

Figure 2. Paw swelling in control, induced arthritis, WJ-MSCs treated rats and 0.5 Gy and 1 Gy irradiated ra 


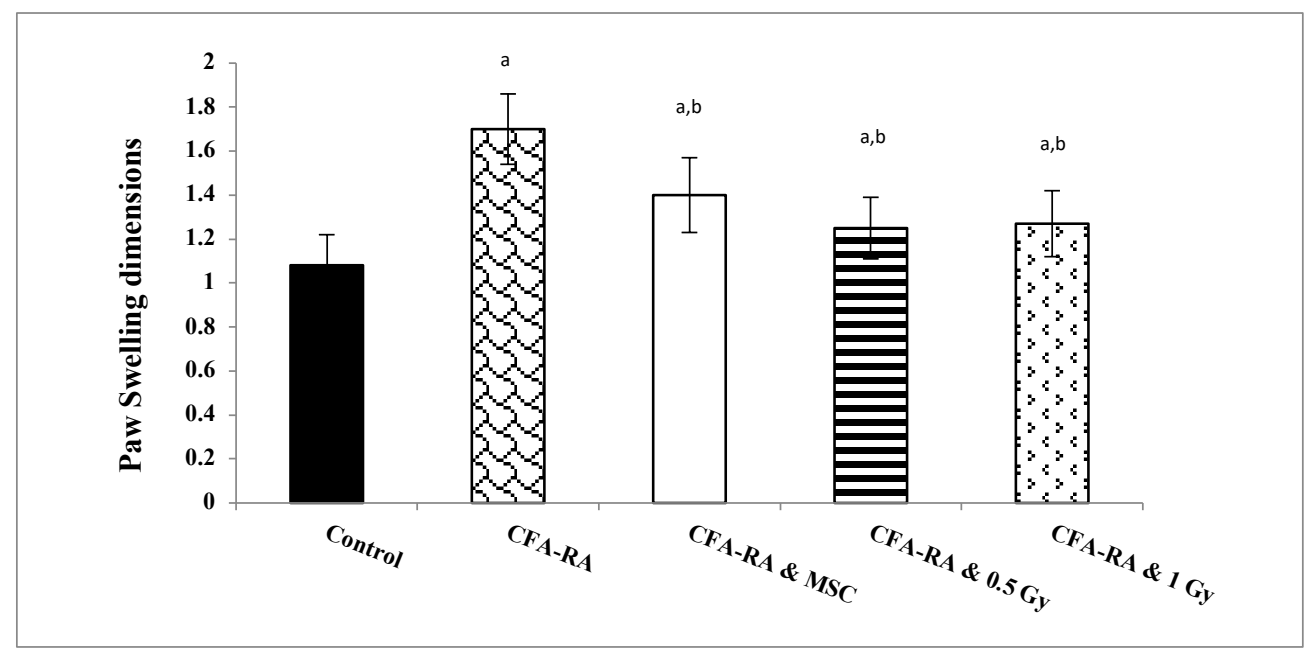

Figure 3: Dimensions of paw swelling in control, induced arthritis, WJ-MSCs treated rats and 0.5 Gy and 1 Gy irradiated rat. (a) Significantly different from the control group; $\mathrm{p} \leq 0.05$. (b) Significantly different from the CFA-RA group; $\mathrm{p} \leq 0.05$.

Table 2. Semi-quantitative scoring for histological findings of the five experimental groups.

\begin{tabular}{ccccc}
\hline Parameters / Score & control & CFA- RA & $\begin{array}{c}\text { CFA- } \\
\text { RA\&MSC }\end{array}$ & $\begin{array}{c}\text { CFA- } \\
\text { RA\&0.5Gy }\end{array}$ \\
\hline Infiltrating mononuclear cells & 0 & 3 & 3 & 2 \\
CFA- \\
Rynovial hyperplasia & 0 & 2 & 2 & 1 \\
\hline Extension of pannus formation & 0 & 3 & 2 & 1 \\
\hline Synovial fibrosis & 0 & 2 & 2 & 2 \\
\hline Cartilage erosion & 0 & 3 & 1 & 0 \\
\hline
\end{tabular}

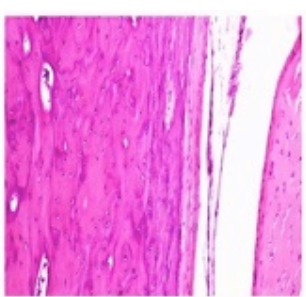

a

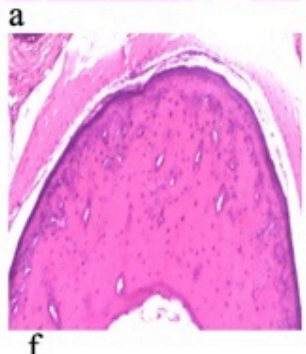

Negative Control

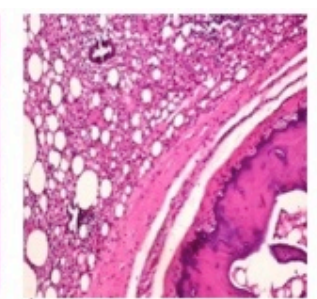
b

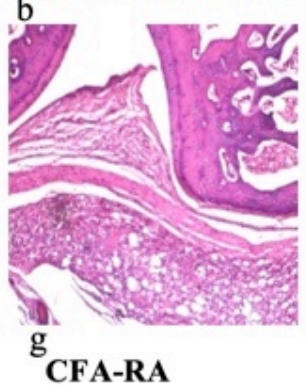

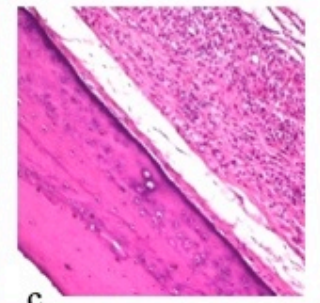

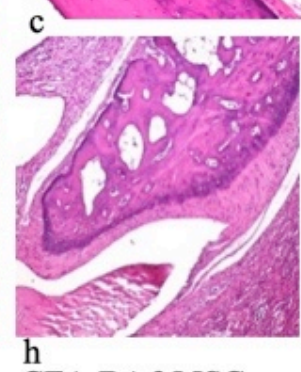

CFA-RA\&MSC

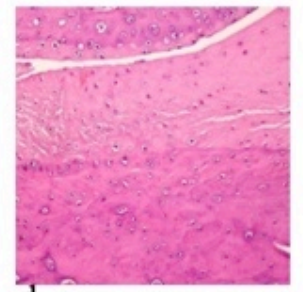

d
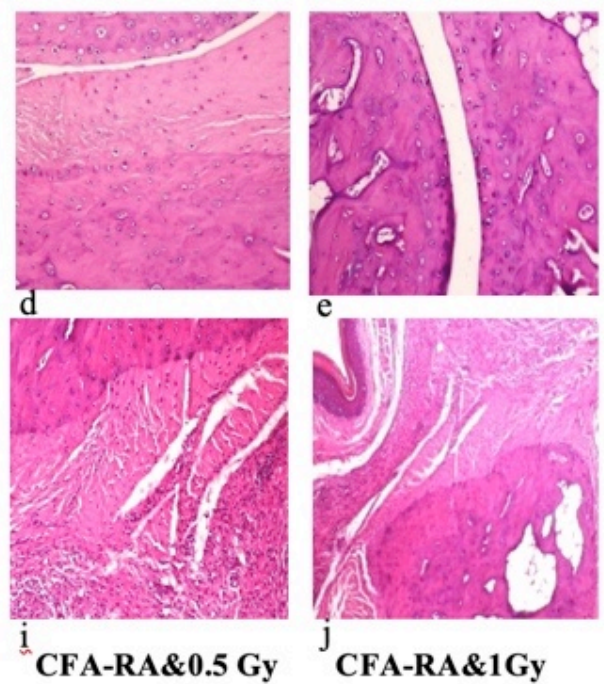

Figure 4. Photomicrographs of paw joint (H\&EX200) showing: (a) normal histological structure of articular surface and synovial membrane (b) normal histologic articular cartilage which consisted of rounded chondrocyte (c) inflammatory cells infiltration and hyperplasia of synovial cells (d) inflammatory granulation tissue and eroding articular cartilage (e) thickening with hyperplasia of synovial membrane epithelial lining (f) collagen fibers between the adipocytes in the stroma and minimal eroded cartilaginous surface (g) moderate number of leukocytic infiltration mainly lymphocytes and macrophages (h) regular and smooth cartilaginous articular surface (i) lower levels of inflammatory cells infiltration mainly lymphocytes and macrophages with well-preserved joint spaces (j) regular smooth cartilaginous surface. 


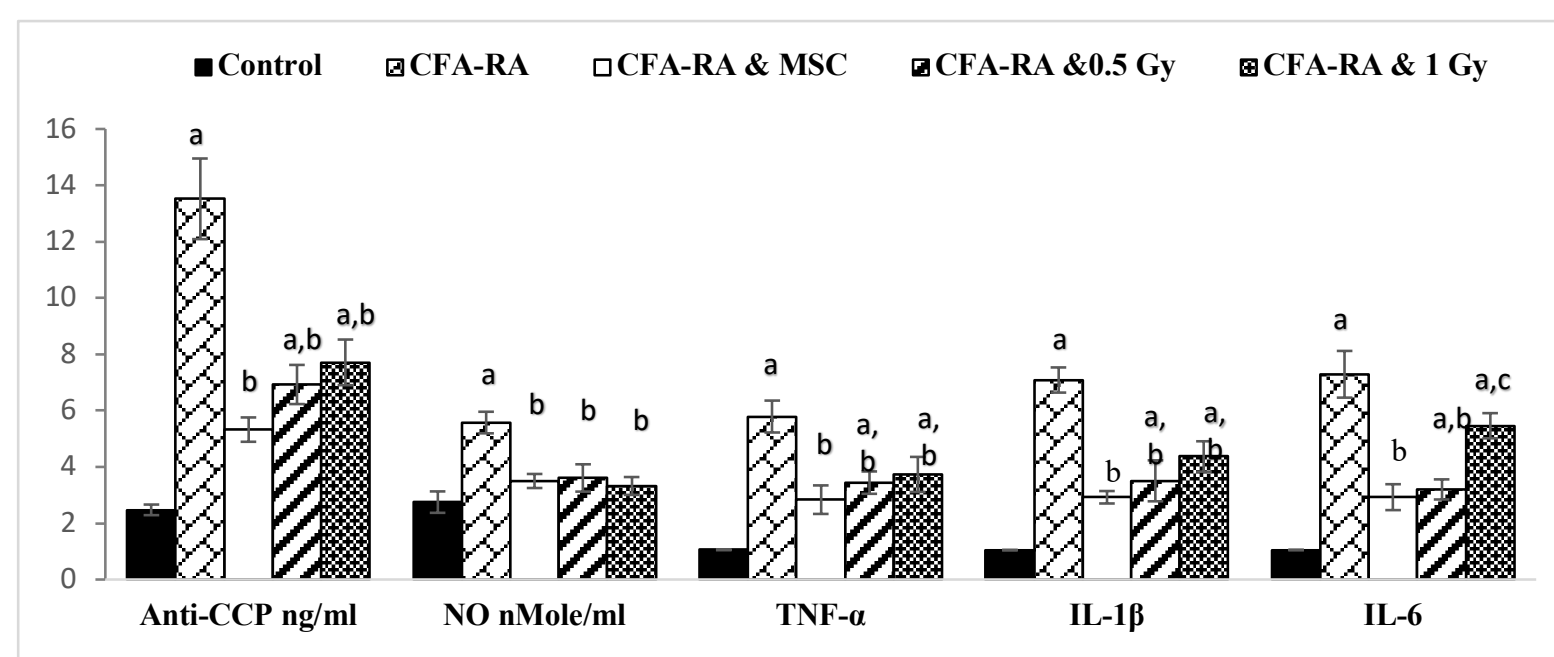

Figure 5. Anti-CCP, plasma NO, TNF- $\alpha$ mRNA, IL-1 $\beta$ mRNA, IL-6 mRNA in control, induced arthritis, WJ-MSCs treated rats and 0.5 Gy and 1 Gy irradiated rats, respectively. (a) Significantly different from the control group; $p \leq 0.05$. (b) Significantly different from the CFA-RA group; $p \leq 0.05$ (c) Significantly different from the CFA-RA \& MSC group; $p \leq 0.05$.

\subsection{Results of gene expression of proinflammatory cytokines in different groups}

TNF- $\alpha$ mRNA, IL-1 $\beta$ mRNA and IL6 mRNA were significantly increased in CFA induced animals compared to control group. Although, these cytokines expression were significantly lowered in WJ-MSC, 0.5 Gy and 1 Gy irradiation treatment groups when compared to experimental arthritis, however there was no significance difference among treated groups regarding TNF- $\alpha$ mRNA and IL- $1 \beta$ mRNA. As for IL-6 mRNA, there was a significant difference between WJ-MSC treated group and 1 Gy irradiated group (Figure 5).

\section{DISCUSSION}

In the present study, success in inducing RA in female rats was achieved by intradermal injection of $0.5 \mathrm{ml}$ of Complete Freund's adjuvant. Full progression of the disease was reached after 10-14 days of adjuvant injection. Further proof for arthritis induction was exhibited by the significant increase in plasma anti-CCP, and NO in CFA treated rats compared to controls. All treated groups, WJ-MSC and individual irradiation with doses 0.5 Gy and 1 Gy demonstrated significant decrease in anti-CCP antibodies compared to CFA induced group, however there was no significant difference between these treated groups. To our knowledge the effect of low dose ionizing radiation on anti-CCP antibodies has not been previously reported neither experimentally nor clinically. Here we report that low dose ionizing radiation ( 0.5 Gy and 1 Gy) showed a significant decrease in the levels of anti-CCP antibodies in experimental adjuvant induced arthritis.
A positive correlation between bone erosion and anti-CCP has been reported ${ }^{30}$. Citrullination is a physiologic pathway that occurs in the apoptotic cell, though it is an inflammatory-dependent condition implicated in autoimmune diseases ${ }^{31}$. When a massive cell death occurs during inflammation, the capacity of the clearance system becomes inadequate to ingest the apoptotic cells. As a result, peptidylarginine deiminase enzymes and citrullinated peptides are released and accumulated inducing loss of tolerance of the immune system in genetically predisposed individuals ${ }^{32}$.

The current study revealed that both WJ-MSC and low dose gamma irradiation with doses of $0.5 \mathrm{~Gy}$ or $1 \mathrm{~Gy}$ were able to reduce swelling, and plasma NO significantly compared to induced arthritic rats. On the other hand, there was no significant difference between the three treated groups regarding swelling and NO levels. Excessive release of NO is implicated in the pathogenesis of RA through enhancing inflammation and vascular permeability. NO can also promote cartilage erosion and cellular damage through oxidative stress pathways ${ }^{33,34}$. Therefore, suppression of nitric oxide levels is associated with alleviation of inflammation and swelling ${ }^{35}$.

RA is characterized by overexpression of proinflammatory cytokines in both synovial tissues and serum $^{36}$. In this research, both treated groups of 0.5 and 1 Gy radiotherapy have significantly reduced the mRNA of pro-inflammatory cytokines TNF- $\alpha$, IL- $1 \beta$ and IL- 6 when compared to arthritic group. These results are consistent with Nakatsukasa et al., $2008^{37}$ who observed significant decrease in serum levels of TNF- $\alpha$ and IL- 6 after irradiation of 0.5 Gy once per 
week for five weeks in collagen induced arthritis. Previous in-vitro studies revealed that low dose radiotherapy can modulate the phenotype of macrophages towards the anti-inflammatory phenotype which is associated with lowering in TNF$\alpha$ and IL- $1 \beta^{38,39}$. Therefore, low dose irradiation can lower the key pro-inflammatory cytokines leading to attenuation of the disease progression. The histological assessment in the present study have shown improvement in the cellular infiltrate, synovial hyperplasia, and cartilage erosion after irradiation in either doses of 0.5 or 1 Gy. Similarly, Deloch et al., $2018^{40}$ also observed attenuation in the inflammatory cell infiltrates and erosive areas after LD-IR of hTNF$\alpha$ tg mice.

Low dose ionizing radiation has been shown to be successful in the treatment of inflammatory and degenerative disorders ${ }^{41}$. During the previous decade, evidence from multiple reports has revealed that lowdose radiotherapy possesses immunomodulatory action due to its impact upon the functional activity of the inflammatory immune cells including endothelial cells, peripheral blood mononuclear cells and activated macrophages. It was reported that LD-RT can suppress polymorphonuclear cell adhesion to endothelial cells due to reduction of adhesion molecules and downregulate inducible nitric oxide synthase generated from activated macrophage, which is associated with the decline in NO production $^{39,42}$. In addition, LD-RT stimulates antiinflammatory cytokines, alters the release of chemokines and cytokines ${ }^{43}$ and induces the expression of antioxidant enzymes ${ }^{44}$.

The results of the present study have demonstrated significant reduction in gene expression of the three pro-inflammatory cytokines after WJMSC treatment as compared to arthritic animals. However, there was no significant difference between the three treated groups regarding lowering gene expression of the three pro-inflammatory cytokines. Related results have been observed by Liu et al. in which ameliorating of the arthritic symptoms in collagen induced arthritis after systemic infusion of human UC-MSC was associated with suppression of pro-inflammatory cytokines TNF- $\alpha$ and IL- $6^{15}$. Regarding histological parameters, treatment with WJ-MSC presented slight improvement in inflammatory cells infiltration, synovial hyperplasia but better improvement in cartilage erosion.

Mesenchymal stem cells are characterized by their regenerative capability and repair through differentiating into lineages of mesenchymal tissues such as chondrocytes, and osteocytes ${ }^{45}$. They can migrate and respond to damaged inflamed microenvironment for repair and recovery ${ }^{46}$.
Furthermore, MSC exhibit immunomodulatory property through its inhibitory effect T- lymphocytes mediated by prostaglandin E2 (PEG2), transforming growth factor $-1 \beta$ (TGB- $\beta 1$ ) and NO along with the suppression of cytokines production ${ }^{47}$. MSC exhibit another special character, which is reduced immunogenicity displayed by lack of acute rejection reaction by the host ${ }^{48}$.

\section{CONCLUSIONS}

Both doses of ( 0.5 Gy and 1 Gy) and WJ-MSC individually attenuated RA effects by significantly decreasing the inflammatory parameters, however, there was no significant difference between them. WJ-MSC and low dose radiation are promising alternative treatments for RA.

Funding: This research did not receive external funding.

Conflicts of Interest: No conflict of interest to be stated.

Ethical Statement: The study protocol was approved by the Research Ethics Committee at Faculty of Pharmacy-Azhar University (86/2016).

Author Contribution: All authors participated in conducting the experiment, analysis of samples and writing the manuscript.

List of Abbreviations: RA: Rheumatoid arthritis; LD-RT: Low-dose radiotherapy; CFA: Complete Freund's Adjuvant; WJ-MSC: Wharton Jelly Mesenchymal Stem Cells; Anti-CCP: Anticyclic citrullinated peptide; NO: Nitric oxide; TNF- $\alpha$ : Tumor necrosis factor- $\alpha$; IL-1 $\beta$ : Interleukin-1 $\beta$; IL6: Interleukin-6; MSCs: Mesenchymal Stromal Cells; UC-MSC: Umbilical Cord Mesenchymal Stem Cells.

\section{REFERENCES}

1. van der Woude D, van der Helm-van AH. Update on the epidemiology, risk factors, and disease outcomes of rheumatoid arthritis. Best Practice \& Research Clinical Rheumatology. 2018;32(2):174-87.

2. Verstappen SM. Rheumatoid arthritis and work: The impact of rheumatoid arthritis on absenteeism and presenteeism. Best Practice \& Research Clinical Rheumatology. 2015;29(3):495-511.

3. Chen W, Wang Q, Ke Y, Lin J. Neutrophil function in an inflammatory milieu of 
rheumatoid arthritis. Journal of immunology research. $2018 ; 3 ; 2018$.

4. Smolen JS, Aletaha D, McInnes IB. Rheumatoid arthritis. Lancet. 2016; 388(10055):2023-38.

5. Phull AR, Nasir B, ul Haq I, Kim SJ. Oxidative stress, consequences and ROS mediated cellular signaling in rheumatoid arthritis. Chemico-Biological Interactions. 2018; 281:121-36.

6. Mateen S, Zafar A, Moin S, Khan AQ, Zubair S. Understanding the role of cytokines in the pathogenesis of rheumatoid arthritis. Clinica Chimica Acta. 2016; 455:161-71.

7. Bui VL, Brahn E. Cytokine targeting in rheumatoid arthritis. Clinical immunology (Orlando, Fla.). 2019; 206:3-8.

8. Matuszewska A, Madej M, Wiland P. Immunological markers of rheumatoid arthritis. Postepy higieny i medycyny doswiadczalnej (Online). 2016; 70:251.

9. Trouw LA, Mahler M. Closing the serological gap: promising novel biomarkers for the early diagnosis of rheumatoid arthritis. Autoimmunity reviews. 2012;12(2):318-22.

10. Rubbert-Roth A. Assessing the safety of biologic agents in patients with rheumatoid arthritis. Rheumatology. 2012;51(suppl_5): v38-47.

11. Sherrer Y. Abatacept in biologic-naive patients and TNF inadequate responders: clinical data in focus. Current medical research and opinion. 2008;24(8):2283-94.

12. Gupta A, El-Amin SF, Levy HJ, Sze-Tu R, Ibim SE, Maffulli N. Umbilical cord-derived Wharton's jelly for regenerative medicine applications. Journal of Orthopaedic Surgery and Research. 2020;15(1):1-9.

13. Watson N, Divers R, Kedar R, Mehindru A, Mehindru A, Borlongan $\mathrm{MC}$, et al. Discarded Wharton jelly of the human umbilical cord: a viable source for mesenchymal stromal cells. Cytotherapy. 2015;17(1):18-24.
14. Weiss ML, Anderson C, Medicetty S, Seshareddy KB, Weiss RJ, VanderWerff I, et al. Immune properties of human umbilical cord Wharton's jelly-derived cells. Stem cells. 2008;26(11):2865-74.

15. Liu Y, Mu R, Wang S, Long L, Liu X, Li R, et al. Therapeutic potential of human umbilical cord mesenchymal stem cells in the treatment of rheumatoid arthritis. Arthritis research \& therapy. 2010;12(6):R210.

16. Sun Y, Kong W, Huang S, Shi B, Zhang H, Chen W, et al. Comparable therapeutic potential of umbilical cord mesenchymal stem cells in collagen-induced arthritis to TNF inhibitor or anti-CD20 treatment. Clin Exp Rheumatol. 2017;35(2):288-95.

17. Rödel F, Fournier C, Wiedemann J, Merz F, Gaipl US, Frey B, et al. Basics of radiation biology when treating hyperproliferative benign diseases. Frontiers in immunology. 2017;8:519.

18. Calabrese EJ, Calabrese V. Low dose radiation therapy (LD-RT) is effective in the treatment of arthritis: animal model findings. International journal of radiation biology. 2013;89(4):287-94.

19. Rödel C, Weiss C. Study of the antiinflammatory effects of low-dose radiation. Strahlentherapie und Onkologie. 2015;191(9):742.

20. Arenas M, Gil F, Gironella M, Hernández V, Jorcano S, Biete A, et al. Anti-inflammatory effects of low-dose radiotherapy in an experimental model of systemic inflammation in mice. International Journal of Radiation Oncology* Biology* Physics. 2006;66(2):560-7.

21. Turk MJ, Breur GJ, Widmer WR, Paulos CM, Xu LC, Grote LA, et al. Folate-targeted imaging of activated macrophages in rats with adjuvant-induced arthritis. Arthritis \& Rheumatism: Official Journal of the American College of Rheumatology. 2002;46(7):1947-55.

22. Fletcher DS, Widmer WR, Luell S, Christen A, Orevillo C, Shah S, et al. Therapeutic administration of a selective inhibitor of nitric oxide synthase does not ameliorate the 
chronic inflammation and tissue damage associated with adjuvant-induced arthritis in rats. Journal of Pharmacology and Experimental Therapeutics. 1998;284(2):714-21.

23. La Rocca G, Anzalone R, Corrao S, Magno $\mathrm{F}$, Loria $\mathrm{T}$, Iacono $\mathrm{ML}$, et al. Isolation and characterization of Oct-4+/HLA-G+ mesenchymal stem cells from human umbilical cord matrix: differentiation potential and detection of new markers. Histochemistry and cell biology. 2009;131(2):267-82.

24. Bancroft JD, Stevens A, Turner DR. Theory and Practice of Histological Techniques. 4th Edn., Churchill, Livingstone, New York, USA. 1996.

25. Barsante MM, Roffê E, Yokoro CM, Tafuri WL, Souza DG, Pinho V, et al. Antiinflammatory and analgesic effects of atorvastatin in a rat model of adjuvantinduced arthritis. European journal of pharmacology. 2005;516(3):282-9.

26. Brenner M, Meng H, Yarlett N, Griffiths M, Remmers E, Wilder R, et al. The non-MHC quantitative trait locus Cia10 contains a major arthritis gene and regulates disease severity, pannus formation and joint damage. Arthritis Rheum. 2005;52(1):32232.

27. Lequin RM. Enzyme immunoassay (EIA)/enzyme-linked immunosorbent assay (ELISA). Clinical chemistry. 2005;51(12):2415-8.

28. Miranda KM, Espey MG, Wink DA. A rapid, simple spectrophotometric method for simultaneous detection of nitrate and nitrite. Nitric oxide. 2001;5(1):62-71.

29. Giulietti A, Overbergh L, Valckx D, Decallonne B, Bouillon R, Mathieu C. An overview of real-time quantitative PCR: applications to quantify cytokine gene expression. Methods. 2001;25(4):386-401.

30. Schellekens GA, Visser H, De Jong BA, Van Den Hoogen FH, Hazes JM, Breedveld FC, et al. The diagnostic properties of rheumatoid arthritis antibodies recognizing a cyclic citrullinated peptide. Arthritis \&
Rheumatism: Official Journal of the American College of Rheumatology. 2000;43(1):155-63.

31. Valesini G, Gerardi MC, Iannuccelli $C$, Pacucci VA, Pendolino M, Shoenfeld Y. Citrullination and autoimmunity. Autoimmunity reviews. 2015;14(6):490-7.

32. Van Venrooij WJ, Van Beers JJ, Pruijn GJ. Anti-CCP antibodies: the past, the present and the future. Nature Reviews Rheumatology. 2011;7(7):391.

33. Ziche M, Morbidelli L. Nitric oxide and angiogenesis. Journal of neuro-oncology. 2000;50(1):139-48.

34. Khojah HM, Ahmed S, Abdel-Rahman MS, Hamza AB. Reactive oxygen and nitrogen species in patients with rheumatoid arthritis as potential biomarkers for disease activity and the role of antioxidants. Free Radical Biology and Medicine. 2016;97:285-91.

35. Nishida K, Doi T, Inoue $H$. The role of nitric oxide in arthritic joints: a therapeutic target? Modern rheumatology. 2000;10(2):63-7.

36. Meyer PW, Hodkinson B, Ally M, Musenge E, Wadee AA, Fickl H, et al. Circulating cytokine profiles and their relationships with autoantibodies, acute phase reactants, and disease activity in patients with rheumatoid arthritis. Mediators of inflammation. 2010;2010.

37. Nakatsukasa H, Tsukimoto M, Ohshima Y, Tago F, Masada A, Kojima S. Suppressing effect of low-dose gamma-ray irradiation on collagen-induced arthritis. Journal of radiation research. 2008;49(4):381-9.

38. Lödermann B, Wunderlich R, Frey S, Schorn C, Stangl S, Rödel F, et al. Low dose ionising radiation leads to a NF- $\kappa$ B dependent decreased secretion of active IL$1 \beta$ by activated macrophages with a discontinuous dose-dependency. International journal of radiation biology. 2012.

39. Deloch L, Fuchs J, Rückert M, Fietkau R, Frey B, Gaipl US. Low-dose irradiation differentially impacts macrophage phenotype in dependence of fibroblast-like 
synoviocytes and radiation dose. Journal of immunology research. 2019;2019.

40. Deloch L, Derer A, Hueber AJ, Herrmann M, Schett GA, Wölfelschneider J, Hahn J, Rühle PF, Stillkrieg W, Fuchs J, Fietkau R. Low-dose radiotherapy ameliorates advanced arthritis in hTNF- $\alpha$ tg mice by particularly positively impacting on bone metabolism. Frontiers in Immunology. 2018; 9:1834.

41. Rödel F, Frey B, Manda K, Hildebrandt G, Hehlgans S, Keilholz L, et al. Immunomodulatory properties and molecular effects in inflammatory diseases of low-dose $\mathrm{x}$-irradiation. Frontiers in oncology. 2012; 2:120.

42. Kern PM, Keilholz L, Forster C, Hallmann R, Herrmann M, Seegenschmiedt MH. Lowdose radiotherapy selectively reduces adhesion of peripheral blood mononuclear cells to endothelium in vitro. Radiotherapy and Oncology. 2000;54(3):273-82.

43. Wunderlich R, Ernst A, Rödel F, Fietkau R, Ott O, Lauber K, et al. Low and moderate doses of ionizing radiation up to 2 Gy modulate transmigration and chemotaxis of activated macrophages, provoke an antiinflammatory cytokine milieu, but do not impact upon viability and phagocytic function. Clinical \& Experimental Immunology. 2015;179(1):50-61.

44. Large M, Hehlgans S, Reichert S, Gaipl US, Fournier C, Rödel C, et al. Study of the antiinflammatory effects of low-dose radiation. Strahlentherapie und Onkologie. 2015;191(9):742-9.

45. Law S, Chaudhuri S. Mesenchymal stem cell and regenerative medicine: regeneration versus immunomodulatory challenges. American journal of stem cells. 2013;2(1):22.

46. Vieira Paladino F, de Moraes Rodrigues J, da Silva A, Goldberg AC. The Immunomodulatory Potential of Wharton's Jelly Mesenchymal Stem/Stromal Cells. Stem cells international. 2019;2019.

47. Ma D, Xu K, Zhang G, Liu Y, Gao J, Tian $\mathrm{M}$, et al. Immunomodulatory effect of human umbilical cord mesenchymal stem cells on $\mathrm{T}$ lymphocytes in rheumatoid arthritis. International immunopharmacology. 2019; 74:105687.

48. Le Blanc K, Tammik C, Rosendahl K, Zetterberg E, Ringdén O. HLA expression and immunologic propertiesof differentiated and undifferentiated mesenchymal stem cells. Experimental hematology. 2003;31(10):890-6. 